\title{
Von Downhill Walking bis Galileo - innovative Rehabilitationsstrategien bei COPD
}

\author{
Neue Trainingsstrategien soll die Rehabilitation von Patienten mit chronisch-obstruktiver Lungenerkrankung be- \\ reichern. Vier davon, die sich ohne großen Aufwand in den Klinikalltag integrieren lassen, stellte ein deutscher \\ Experte auf dem ERS-Kongress in Mailand vor.
}

Zum Downhill Walking wird nicht mehr benötigt als ein übliches Laufband, das mithilfe einer etwa 200 Euro teuren Vorrichtung mit einem Neigungswinkel von 10 Grad schräg gestellt wird. Wenn ein Patient darauf marschiert, sinkt der Sauerstoffverbrauch um $17 \%$ im Vergleich zum Laufen in der Ebene, die Ventilation nimmt um $9 \%$ ab. Zugleich steigt aber die muskuläre Anstrengung, die Muskeln ermüden schneller.

„Das ergibt einen guten Trigger für die Belastungskapazität bei signifikant weniger Dyspnoe“, erläuterte Dr. Rainer Glöckl, von der Schön Klinik Berchtesgadener Land im Schönau am Königssee. In einer Studie an 23 COPD-Patienten konnte gezeigt werden, dass fast alle Patienten nach Downhill Walking im Sechsminutengehtest mehr als 30 Meter zugelegt hatten und zwei Drittel sich im Atemnot-Score mMRC („modified Medical Research Council“) um mindestens einen Punkt verbessert hatten. Patienten, die in der Ebene trainiert hatten, schnitten deutlich schlechter ab. Nebenwirkungen gab es kaum, nur zwei Patienten entwickelten eine Sehnenscheidenentzündung am M. tibialis anterior.

\section{Einbeiniges Radeln}

Auf einem ähnlichen Konzept - effektives Training mit weniger Atemnot - basiert die Idee, Patienten einbeinig radeln zu lassen. Dabei sind weniger Muskeln beteiligt, also fällt der Sauerstoffbedarf niedriger aus als beim normalen Ergometer-Training. Die Patienten lernen schnell, nur ein Bein $\mathrm{zu}$ benutzen, und schätzen das einfache und kardiopulmonal weniger belastende Training.

Nach Einschätzung von Glöckl eignet sich diese Methode für Patienten mit fortgeschrittener COPD, ausgeprägter Dyspnoe und Neigung zur Entsättigung besonders gut. Seine Empfehlung: mit 40 bis $50 \%$ der Maximalleistung beginnen und pro Bein 10 bis 15 Minute trainieren lassen. Eine spezielle Ausstattung ist nicht unbedingt vonnöten. Es kann aber sinnvoll sein, das inaktive Bein auf einen Stuhl oder Kasten ablegen zu lassen.

\section{Nicht invasive Beatmung während des Trainings}

Auch die dritte Innovation zielt darauf ab, Patienten das Training zu erleichtern, indem ihnen die Dyspnoe genommen wird.

Die nicht invasive Beatmung (NIV) während des Trainings „hat momentan noch experimentellen Status, räumte Glöckl ein. Das Konzept ist, die Atemarbeit zu erleichtern, sodass unter anderem der Blutfluss zur Versorgung der Skelettmuskulatur gesteigert wird. Beim europäischen Pneumologie-Kongress stellte Glöckls Arbeitsgruppe eine eigene Crossover-Studie vor, an der 15 Patienten mit fortgeschrittener COPD (FEV ${ }_{1}$ im Schnitt unter $20 \%$ v. S., pCO $51 \mathrm{mmHg}$ ) teilgenommen haben.

Unter NIV konnten die Patienten signifikant länger auf dem Ergometer trainieren als ohne Beatmung. „Das Training mit NIV ist gewöhnungsbedürftig“, räumte der Pneumologe ein. Es könnte aber vor allem hyperkapnischen Patienten helfen, sicher und mit höherer Intensität zu trainieren.

Das Verfahren ist zeit-, ressourcen- und personalaufwendiger als die beiden ersten und dürfte auch deshalb ausgewählten $\mathrm{Pa}$ tientengruppen vorbehalten bleiben.

Das Ganzkörper-Vibrationstraining (WBVT) mit der Galileo-Plattform, ursprünglich für Osteoporose und andere Erkrankungen des Bewegungsapparats entwickelt, erreicht inzwischen viele Bereiche der Medizin, in denen eine Bewegungstherapie indiziert ist auch die COPD. Dabei steht der Patient auf einen Platte, die Schaukelbewegungen in hoher Frequenz erzeugt. Dadurch werden unwillkürliche Kontraktionen der Muskulatur induziert.

Von acht Studien, die es zur COPD gibt, sind sieben zugunsten des WBVT ausgegangen. Nur in einer wurde im Sechsminutengehtest kein Unterschied zur Kontrollgruppe gesehen. Es gibt sogar schon eine Metaanalyse, die sehr gute Resultate für die körperliche Leistungsfähigkeit bei geringen Nebenwirkungen zeigt.

Warum das WBVT COPD-Patienten nutzt, ist nicht ganz einfach zu ergründen. Kardiopulmonale Effekte scheinen dafür ebenso wenig verantwortlich $\mathrm{zu}$ sein wie eine verbesserte Muskelkraft. Am ehesten kommt eine bessere neuromuskuläre Koordination infrage, so Glöckl. Die besten Effekte werden tatsächlich bei Patienten erzielt, die kaum körperlich belastbar sind und eine schlechte Balancekoordination zeigen.

Manuela Arand

Ganzkörper-Vibrationstaining bringt offenbar auch was bei COPD. Warum?
Quelle: Kongress der European Respiratory Society, Mailand, 9.-13.9.2017 